\title{
PSO-CCO_MIMO-SA: A particle swarm optimization based channel capacity optimization for MIMO system incorporated with smart antenna
}

\author{
Shivapanchakshari T. G. ${ }^{1}$, H. S. Aravinda ${ }^{2}$ \\ ${ }^{1}$ Department of Electronics and Communication Engineering, \\ Cambridge Institute of Technology, Visvesvaraya Technological University, India \\ ${ }^{2}$ Department of Electronics and Communication Engineering, J.S.S. Academy of Technical Education, India
}

\begin{tabular}{l}
\hline \hline Article Info \\
\hline Article history: \\
Received Oct 24, 2019 \\
Revised May 20, 2020 \\
Accepted Jun 2, 2020 \\
\hline
\end{tabular}

\section{Keywords:}

Channel capacity

MIMO system

PSO

Signal to noise ratio (SNR)

Smart antenna

\begin{abstract}
With the radio channels physical limits, achieving higher data rate in the multi-channel systems is been a biggest concern. Hence, various spatial domain techniques have been introduced by incorporating array of antenna elements (i.e., smart antenna) in recent past for the channel limit expansion in mobile communication antennas. These smart antennas help to yield the improved array gain or bearm forming gain and hence by power efficiency enhanmaent in the channel and antenna range expansion. The use of smart antenna leads to spatial diversity and minimizes the fading effect and improves link reliability. However, in the process of antenna design, the proper channel modelling is is biggest concern which affect the wireless system performance. The recent works of MIMO design systems have discussed the issues in number of antenna selection which suggests that optimization of MIMO channel capacity is required. Hence, a Particle Swarm Optimization based channel capacity optimzation for MIMO system incorporated with smart antenna is introduced in this paper. From the outcomes it is been found that the proposed PSO based MIMO system achieves better convergenece speed which results in better channel capacity.
\end{abstract}

Copyright $@ 2020$ Institute of Advanced Engineering and Science. All rights reserved.

\section{Corresponding Author:}

Shivapanchakshari T. G.,

Research Scholar, Department of Electronics and Communication Engineering,

Cambridge Institute of Technology,

Visvesvaraya Technological University, Belagavi, India

Email: tgsresearch2013@gmail.com

\section{INTRODUCTION}

The channel capcity has became an majorly considerable paramerter in todays and upcoming wireless systems for the communication [1]. Generally, the channel capacuty of an fading channel can be referred as the sum of all the narrow band flat fading channel capacities at every discrete frequency points [2]. Hence, various radio techniques have been evolved in recent past where wideband technique has come up with better data communication rate for the short range wireless system [3]. The reason behind the higher data communication with the wideband technique is that it exhibits highe bandwidth of $3.1 \mathrm{GHz}$ to $10.6 \mathrm{GHz}[4,5]$. The channel capacity of MIMO system in a wirelss communication system is higher than the conventional system under multi-path environment scenario [6]. Most of the existing systems ave discussed the channel capacity of the MIMO system but lack with researches associated with the smart antenna and MIMO system channel capacity optimization mechanims [7, 8]. The previous work of Shivapanchakshari and $[9,10]$ have discussed about the complexity impprovement as well as performance enhancement for the smart antenna system. This paper aims to extend the work of $[9,10]$ with respect to channel capacity optimization and array pattern in MIMO and Smart antenna system by introducing an 
optimizer based on particle swarm optimization (PSO technique). In this, paper the cost function is considered with PSO for non-smooth and discontinous anttenna pattern. The manuscript is catogorized into different sections which includes: review of exisiting researches intended to enhance the channel capacity if MIMO-smart antenna by adapting various methodologies (section 2) along with research gap and problem statement. The adapted research methodology (section 3) along with the algorithm implementation is discussed in section 4 . The analysis of the simulation results are discussed in section-5 while the comclusive points and future scope of the manuscript is highlighted in section 6 .

\section{REVIEW OF LITERATURE AND RESEARCH GAP}

This section discusses various researches towards perfroamnce enhancement in the wireless communication with the MIMO systems. A recent work of Piazza et al., [11], have intvestigated the performance scenarios in broadband and narrowband MIMO systems by introducing the bi-port reconfigurable patch antenna. The study has considered field measurements from the indoor environment for channel capacity at noth communication ends. The outcomes were analysed with nonline of sight and line of sight cases of polarization and found that the techniques can be applicable to both broadband and narrowband MIMO systems. A work of Nielsen et al., [12] MIMO channel capacity measurement for the smartphone under data mode operation. The channel measurement is carried out for different frequency bands from the base station in free space with 12 users. The outcomes are measured for random channel capacities in terms of outage capacity. The limitation factor of this [12] work is that it has not conducted the performance anlysis with the existing research. An interesting work of Chang and $\mathrm{Hu}$ [13] has discussed the design prospectives of the smart antenna and their implemention in the advanced communication system. The work suggests that the use of the smart antenna in the communication system enhances the network capacity. The author has the expanded his ideas with the different smart anttena analysis, multibeam MIMO antenna etc. The performance analysis of the handheld MIMO channel under noise and interference conditon is conducted in Plicanic et al., [14] by using 3-antenna (multiple) configuration. The communication performance is measured by considering the multipath channel under interference and noise condition which obtains the improved channel capacity. Rosas and Oberli [15] have introduced an attractive MIMO technique to minimize the power consumption where the MIMO channel capacity is improved with large diversity gain and optimal power usage. Shelzinger et al. [16] have presented a Gaussian channel capacity for MIMO under power contrained condition and achieved the secrecy capacity improvement.

The recent work of Leftah and Alminshid [17] have presented a discrete transform based MIMO-OFDM system under channel fading condition and used different amplitude modulation techniques. The performance of the system is been measured with Bit Error Rate (BER) and system channel capacity. From the comparison with the existing system it is been observed that the discrete transform based MIMO-OFDM offers better channel capacity than the conventional MIMO-OFDM systems. Similar kind of work is observed in Duong [18] (improved channel capacity), Singal and Kedia [19] (performance analysis of MIMO antenna selection mechanism).

Also, other researches were tried to imporove the MIMO channel capcity using different techniques i.e., Wong et al., [20], Xu et al., [21], Yang Li et al., [22], Yixin Li et al., [23, 24], Zhang et al., [25], Guo et al., [26], Shafie et al. [27], Jiang et al. [28, 29], Wang et al. [30], Wong et al. [31], Akhtar and Gesbert [32], Ho et al., [33]. From the above research survey, it is been observed that most of the researches were considered MIMO and antenna techniques separately for wireless communication performance enhancement and very rare researches were observed with the combination of both. Also, it is been observed that least number of works were focused on the optimization of channel capacity. Thus, the problem statement is "need of optimization technique which can offer optimized channel capacity in MIMO system at different scenario".

\section{PROPOSED MODEL OF CHANNEL CAPACITY OTIMIZATION}

The proposed system of PSO based channel capacity optimization (CCO) for MIMO incorporated smart antenna (SA) (PSO-CCO_MIMO-SA) aims to perform the optimization of channel capacity which is rarely addressed in the research domain. The proposed model is the conitination of [10] work. The MIMO system uses Binary Phase Shift Keying (BPSK) modulation technique for the BER calculation. The MIMO system of 2-transmitter (Tx) and 2-Reciever (2-Rx) uses a Rayleigh fading channel for Zero Forcing Equalization (ZFE) which is a linear optimization algorithm used for inverse of channel frequency response. The system is composed of 'Ns' number of symbols/bits, multiple number of Energy per Bit $\left(\mathrm{E}_{\mathrm{b}}\right)$ to noise power spectral density $\left(\mathrm{N}_{0}\right)$ ratio $\left(\mathrm{E}_{\mathrm{b}} / \mathrm{N}_{0}\right)$. The channel and noise are added and applied PSO to the transmitter unit before forwarding the symbols to the receiver. The consideration of the PSO at the transmitter end helps 
to bring better CCO. The Receiver forms a matrix for the ZFE and formats the received symbols to perform the equalization. The receiver takes decision on decoding of the received signal. Finally, the computation of the errors (BER) can be computed. The architectural model of the PSO-CCO_MIMO-SA is given in Figure 1. The design is introduced with the synthesis of the array pattern to maximize the capacity of the channel performance. The system is analysed at scenario-1 (LOS) and scenario-2 (NLOS), where the mean excess delay and root mean square (RMS) delay is adjusted to syntehss of the antenna pattern. However, the synthesis leads to excitation problem in the optimization. Hence, the PSO alfotithm is used to perform the regulation of the antenna feed length and achieve better channel capacity. Thus, the signal to noise ratio (SNR) will be improved and BER will be reduced. The system performance can be analysed with respect to scenario-1 (LOS) and scenario-2 (NLOS). The LOS scenario is the direct radio wave propagation between two antennas where the propagation loass is almost similar to the free space impact while NLOS scenario is the improper or partially obstracted wave propagation in which the transmission quality get affected.

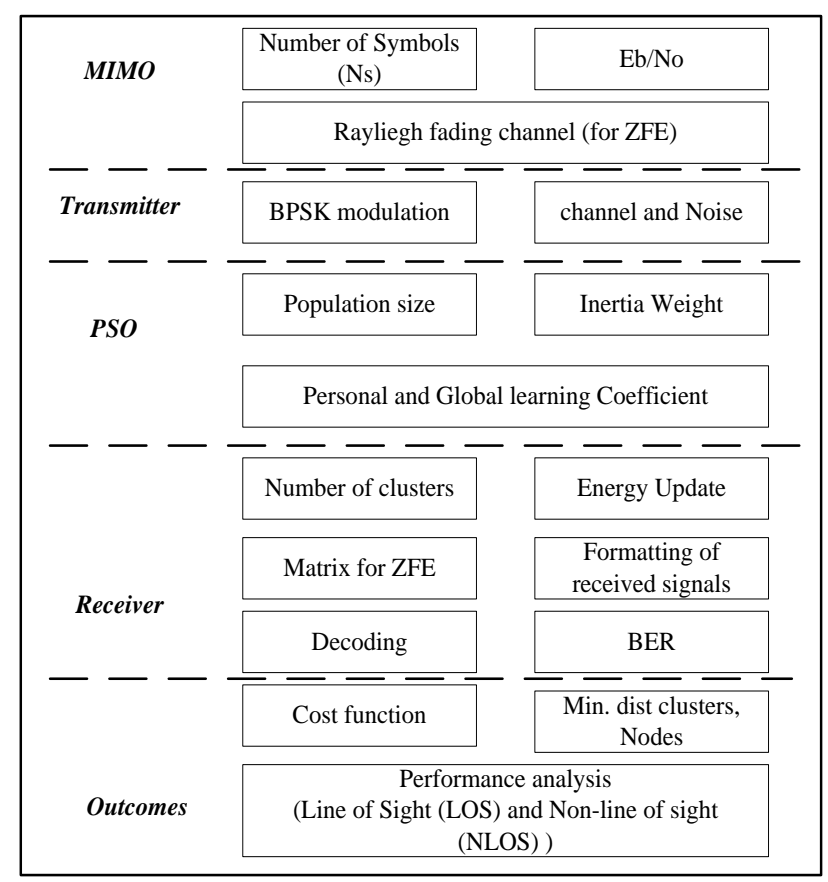

Figure 1. Architecture of the PSO-CCO_MIMO-SA

The channel capacity of the MIMO channel is computed by considering the deterministic matrix (Dm) of Tx, Rx. The capacity of the 'Dm' is computed by performing the channel decomposition into parallel and scalar white Gaussian sub channels. The element of Dm (real numbers as negative and others as zero). The MIMO system is able to process the signal at both $\mathrm{Tx}$ and $\mathrm{Rx}$ end and produces the cluster of the received signal at higher capacity. The PSO algorithm is a population-based algorithm and is mainly used for the global optimization problems. The PSO considers only rare parameter adjustement at rapid speed. The use of PSO found in the real numbers than parameter coding and is works on natural bird flocking features. Hence, the proposed model considers PSO for optimization where the antenna excitation voltage and array elements feed length is regulated to get the optimal radiation pattern as well as thr enhanced channel capacity. The PSO algorithm is also used in the synthesis process by which the cost function $(C f)$ can be minimized and is defined as the inverse of channel capacity. The next section discussed the algorithm implementation.

\section{ALGORITHM IMPLEMENTATION}

The algorithm for the proposed PSO-CCO_MIMO-SA is designed by considering the PSO algorithm in the MATLAB. The algorithm is initialized by using number of samples (Ns), multiple values of Eb/N0, number of Tx, Number of Rx (step-1, 2). For the same length of Eb/N0, the bits 0,1 are generated with equal 
probability (Step-3). Furhter, the BPSK modulation scheme is applied (step-4). The Tx and Rx are grouped in a matrix. The Rayleigh channel and white gausian noise is added to the tranmistted symbol (Step-5). The PSO is applied to the transmitted symbol to get the better optimization in the channel capacity, where the number of iterations (Ni), number of population (Np) or swarm size, intertial weight (Iw), damping of Iw (Iwd), PLC, GLC are defined. The numbers of clusters (Nc) are formed in the PSO based on the population size and path. The decision veriable (Dv) size and matrix formed based on the generated matrix. Based on the defined velocity of the optimization the PSO evaluates the Global best $(\mathrm{Pb})$ and Personal best $(\mathrm{Pb})$ solution for the selecting the sendor randomly. Finally, the energy computation is performed at PSO and updated (Step-6). Once, the implementationof the PSO is done, the Rx will form a ZFE matrix (Step-7) on the basis of cluster of Tx, Rx and this matix is used to format the received symbols to perform the symbol equalization (Step-8). Later, the Rx will take a decision to decode the received symbol to get in original format (Step-9). The channel capacity is meansured by computing the BER in the Rx end (Step-10).

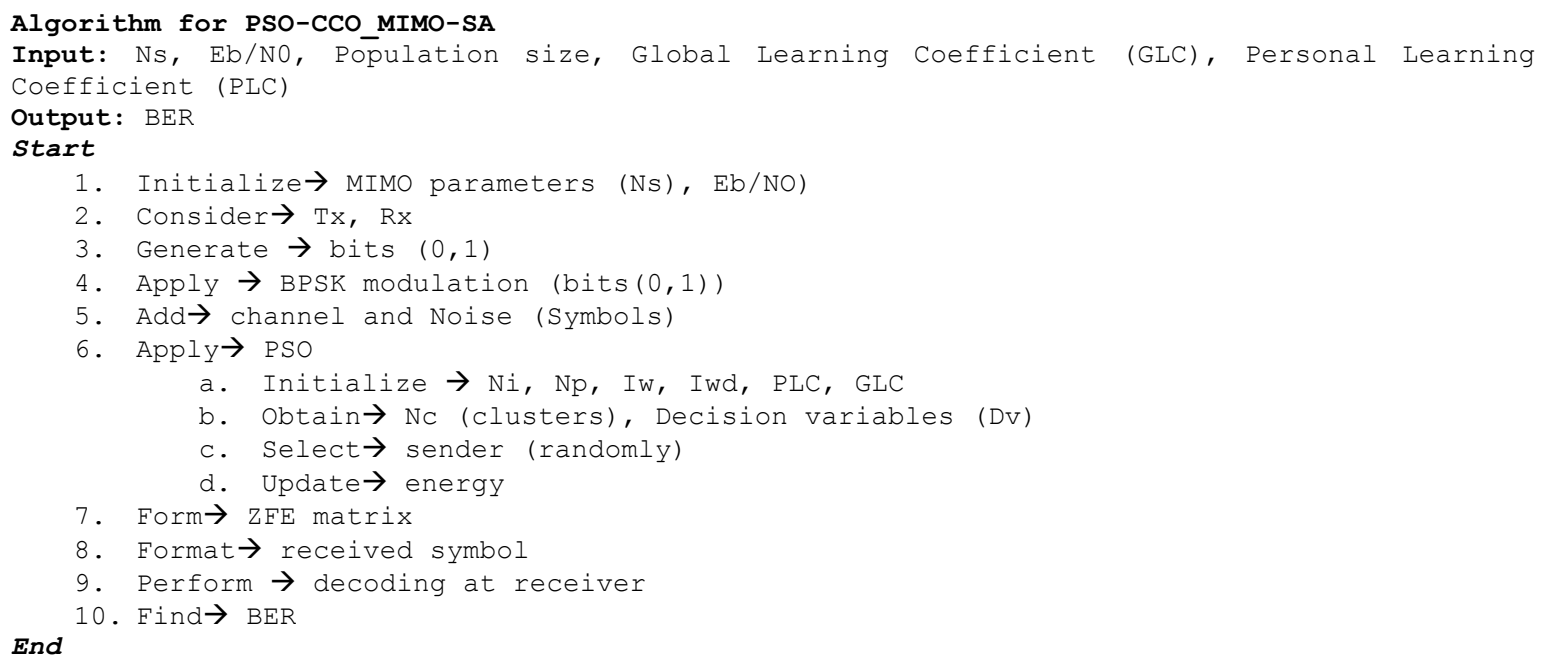

The algorithm with the antenna array for channel capacity enhancement in the MIMO-SA using is described above where synthesis problem is reformulated as the optimization problem in which the cost function is minimized by adjusting each antenna feed length. The next section discusses on the results analysis of PSO-CCO_MIMO-SA model.

\section{RESULTS ANALYSIS}

The modelling of the proposed PSO-CCO_MIMO-SA model is performed over MATLAB software. The value of the design parameters is given in Table 1 . The model is anlyzed in two scenarions (scenario- 1 ) LOS and (scenario-2) NLOS. The mean exess delay (0.25 and 2) and RMS delay (0.49 and 2.02) respectively set for scenario-1and scenario-2. The oprimization process is conducted for 0:10 i.e., $11 \mathrm{~Eb} / \mathrm{N} 0$ values. The remaining optimization for each Eb/N0 value is given in Figure 2. The following Figure 3 gives the variation in the BER with respect to different values of $\mathrm{Eb} / \mathrm{N}$ ) where the BPSK modulation is considered for different set of Tx and Rx. The ZFE with Rayligh channel is compared at drawn to measure the error Rate of $(1 \times 1,1 \times 2$ and 2x2) Tx and Rx set.

Table1. Design parameters value

\begin{tabular}{ll}
\hline Type & Value \\
\hline Symbols (Ns) & $10^{6}$ \\
Eb_N0 & $0: 10$ \\
Transmitter (Tx) & 2 \\
Reciever (Rx) & 2 \\
Iterations (Ni) & 1000 \\
Ppulation (Np) & 100 \\
Inertia weight (Iw) & 1 \\
Damping (Iwd) & 0.99 \\
PLC & 1.5 \\
GLC & 2.0 \\
\hline
\end{tabular}




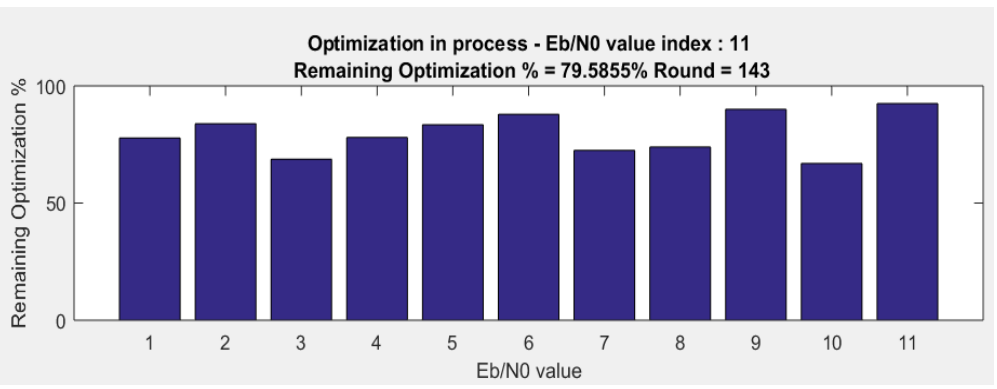

Figure 2. Remaning optimation \% with respect to Eb/N0 value

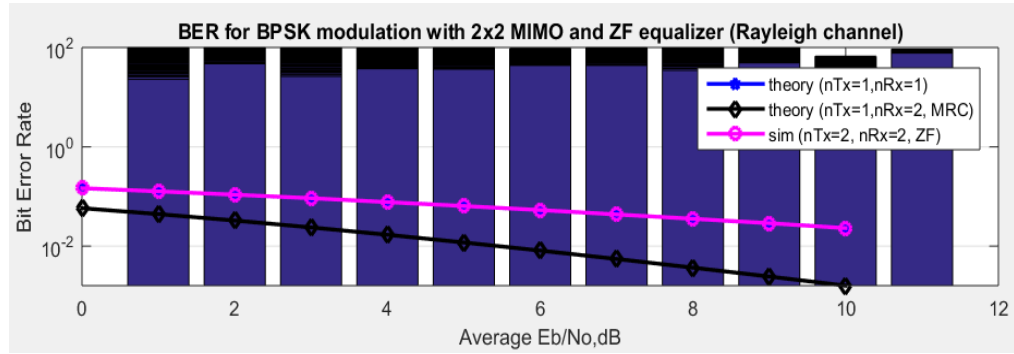

Figure 3. BER Vs Average Eb/N0 at different Tx and Rx set

The performance analysis of the proposed PSO based MIMO system is performed by comparing it with the existing genetic algorithm (GA) based MIMO in both LOS (scenario-1) and NLOS (scenario-2) scenario by considering channel capacity and SNR as performance parameter. Figure 4 gives the channel capacity Vs SNR of $2 \times 2$ MIMO systems at scenario-1 and scenario-2 where the SNR is the average transmitting power to noise ratio. From the Figure 4, it is been obsereved that the channel capacity of proposed PSO based MIMO system is gradually increasing than GA based MIMO as it minimizes the fading and the multipath effects. Also, the synthesized array pattern of the antenna has optimized the processing gain to the receiver. Similarly, the Figure 5 gives the channel capacity Vs SNR of SISO, MIMO and proposed PSO based MIMO systems at scenario-1 and scenario-2. In both the scenarios the proposed PSO based MIMO system channel capacity is better than the other communication systems.

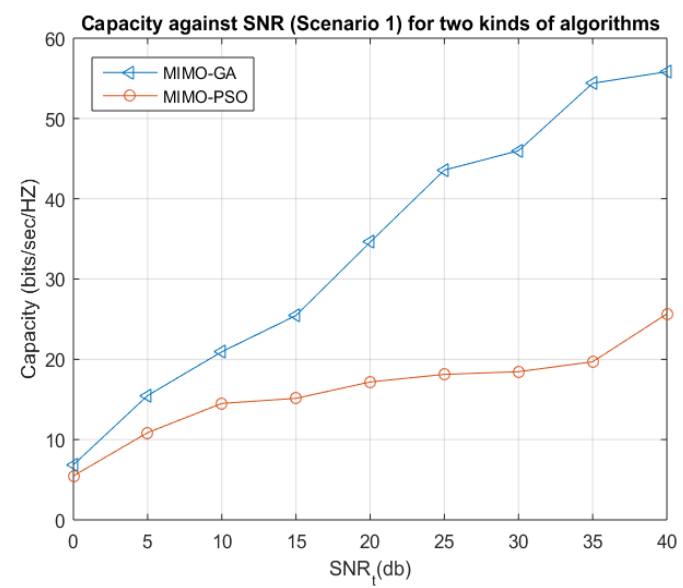

(a)

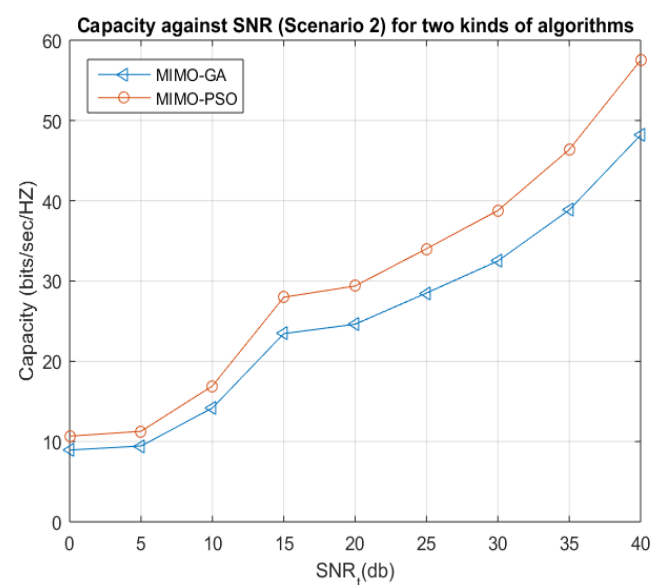

(b)

Figure 4. Channel capacity Vs SNR at different scenarios (LOS and NLOS), (a) channel capacity Vs SNR @ Scenario-1, and (b) channel capacity Vs SNR @ Scenario-2 


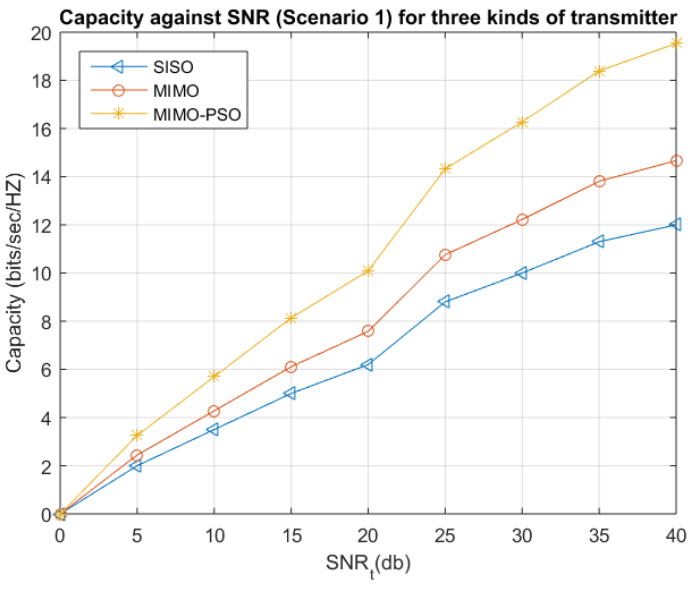

(a)

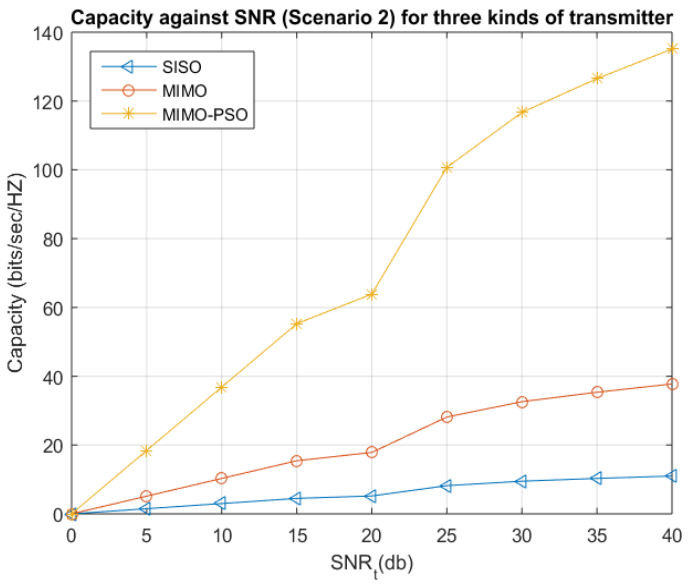

(b)

Figure 5. Channel capacity Vs SNR at different communication systems (SISO, MIMO and Proposed PSO based MIMO), (a) channel capacity Vs SNR @ Scenario-1, (b) channel capacity Vs SNR @ Scenario-2

\section{CONCLUSION}

This paper introduces a PSO-CCO_MIMO-SA system where MIMO system uses binary phase shift keying (BPSK) modulation technique for the BER calculation. The MIMO system of 2-transmitter (Tx) and 2-Reciever (2-Rx) uses a Rayleigh fading channel for zero forcing equalization (ZFE) which is a linear optimization algorithm used for inverse of channel frequency response. The design is introduced with the synthesis of the array pattern to maximize the capacity of the channel performance. The system is analysed for the channel capacity Vs SNR of 2x2 MIMO systems scenario-1 (LOS) and scenario-2 (NLOS) with GA and SISO, MIMO and proposed PSO based MIMO systems. From the outcomes it is been found that the proposed PSO based MIMO system achieves better convergenece speed which results in better channel capacity.

\section{REFERENCES}

[1] Mo, Jianhua, and Robert W. Heath. "Capacity analysis of one-bit quantized MIMO systems with transmitter channel state information," IEEE transactions on signal processing, vol. 63, no. 20, pp. 5498-5512, 2015.

[2] Yankov, Metodi P., et al. "Approximating the constellation constrained capacity of the MIMO channel with discrete input," IEEE International Conference on Communications, 2015.

[3] Erseghe, T., "Capacity of UWB impulse radio with single-user reception in Gaussian noise and dense multipath," IEEE Transactions on Communications, vol. 53, no. 8, pp. 1257-1262, 2005.

[4] Yang, L., Giannakis, G. B., "Ultra-wideband communications: an idea whose time has come," IEEE Signal Process. Mag., vol. 21, no. 6, pp. 26-54, 2004.

[5] Roy, S., Foerster, J. R., Srinivasa Somayazulu, V., Leeper, D. G., "Ultrawideband radio design: The promise of high-speed, short-range wireless connectivity," Proc. IEEE, vol. 92, no. 2, pp. 295-311, 2004.

[6] Hu, Z., et al., "Filter-free optically switchable and tunable ultrawideband monocycle generation based on wavelength conversion and fiber dispersion," IEEE Photonics Technol. Lett., vol. 22, no. 1, pp. 42-44, 2010.

[7] Adinoyi, A., Yanikomeroglu, H., "Practical capacity calculation for time-hopping ultra-wide band multiple-access communications," IEEE Communications Letters, vol. 9, no. 7, pp. 601-603, 2005.

[8] Ramı'rez-Mireles, F., "On the capacity of UWB over multipath channels," IEEE Communications Letters, vol. 9, no. 6, pp. 523-525, 2005.

[9] Shivapanchakshari, T. G., and H. S. Aravinda, "An efficient mechanism to improve the complexity and system performance in OFDM using switched beam smart antenna (SSA)," Computer Science On-line Conference, pp. 60-66, 2019.

[10] Shivapanchakshari, T. G., and H. S. Aravinda, "Adaptive Resource Allocation using various Smart Antenna Techniques to maintain better System Performance," International Journal of Engineering and Advanced Technology (IJEAT), vol. 8, no. 5S, pp. 262-265, May 2019.

[11] D. Piazza, et al., "Experimental Analysis of Pattern and Polarization Reconfigurable Circular Patch Antennas for MIMO Systems," IEEE Transactions on Vehicular Technology, vol. 59, no. 5, pp. 2352-2362, 2010.

[12] J. Ø. Nielsen, et al., "User Influence on MIMO Channel Capacity for Handsets in Data Mode Operation," IEEE Transactions on Antennas and Propagation, vol. 60, no. 2, pp. 633-643, 2011.

[13] D. Chang and C. Hu, "Smart Antennas for Advanced Communication Systems," Proceedings of the IEEE, vol. 100, no. 7, pp. 2233-2249, 2012. 
[14] V. Plicanic, H. Asplund and B. K. Lau, "Performance of Handheld MIMO Terminals in Noise- and InterferenceLimited Urban Macrocellular Scenarios," IEEE Transactions on Antennas and Propagation, vol. 60, no. 8, pp. 3901-3912, 2012.

[15] F. Rosas and C. Oberli, "Impact of the Channel State Information on the Energy-Efficiency of MIMO Communications," IEEE Transactions on Wireless Communications, vol. 14, no. 8, pp. 4156-4169, 2015.

[16] N. Shlezinger, D. Zahavi, Y. Murin and R. Dabora, "The Secrecy Capacity of Gaussian MIMO Channels With Finite Memory," IEEE Transactions on Information Theory, vol. 63, no. 3, pp. 1874-1897, 2017.

[17] Leftah, Hussein A., and Huda N. Alminshid, "Channel capacity and performance evaluation of precoded MIMOOFDM system with large-size constellation," International Journal of Electrical and Computer Engineering (IJECE), vol. 9, no. 6, pp. 5024-5030, 2019.

[18] Ai, D. Huu, "Average Channel Capacity of Amplify-and-forward MIMO/FSO Systems Over Atmospheric Turbulence Channels," International Journal of Electrical and Computer Engineering (IJECE), vol. 8, no. 6, pp. 4334-4342, 2018.

[19] Singal, Anuj, and D, Kedia, "Performance analysis of antenna selection techniques in mimoofdm system with hardware impairments: energy efficiency perspective," International Journal of Electrical and Computer Engineering (IJECE), vol. 8, no. 4, pp. 2272-2279, 2018.

[20] K. Wong, C. Tsai and J. Lu, "Two asymmetrically mirrored gap-coupled loop antennas as a compact building block for eight-antenna MIMO array in the future smartphone," IEEE Transactions on Antennas and Propagation, vol. 65, no. 4, pp. 1765-1778, 2017.

[21] Z. Xu, Y. Sun, Q. Zhou, Y. Ban, Y. Li and S. S. Ang, "Reconfigurable MIMO Antenna for Integrated-MetalRimmed Smartphone Applications," IEEE Access, vol. 5, pp. 21223-21228, 2017.

[22] M. Li, Z. Xu, Y. Ban, C. Sim and Z. Yu, "Eight-port orthogonally dual-polarised MIMO antennas using loop structures for 5G smartphone," IET Microwaves, Antennas \& Propagation, vol. 11, no. 12, pp. 1810-1816, 2017.

[23] Y. Li, C. Sim, Y. Luo and G. Yang, "Multiband 10-Antenna Array for Sub-6 GHz MIMO Applications in 5-G Smartphones," IEEE Access, vol. 6, pp. 28041-28053, 2018.

[24] Y. Li, et al., "High-Isolation 3.5 GHz Eight-Antenna MIMO Array Using Balanced Open-Slot Antenna Element for 5G Smartphones," IEEE Transactions on Antennas and Propagation, vol. 67, no. 6, pp. 3820-3830, 2019.

[25] Y. Zhang, S. Yang, Y. Ban, Y. Qiang, J. Guo and Z. Yu, "Four-feed reconfigurable MIMO antenna for metal-frame smartphone applications," IET Microwaves, Antennas and Propagation, vol. 12, no. 9, pp. 1477-1482, 2018.

[26] J. Guo, L. Cui, C. Li and B. Sun, "Side-Edge Frame Printed Eight-Port Dual-Band Antenna Array for 5G Smartphone Applications," IEEE Transactions on Antennas and Propagation, vol. 66, no. 12, pp. 7412-7417, 2018.

[27] A. E. Shafie, H. Chihaoui, R. Hamila, N. Al-Dhahir, A. Gastli and L. Ben-Brahim, "Impact of Passive and Active Security Attacks on MIMO Smart Grid Communications," IEEE Systems Journal, vol. 13, no. 3, pp. 2873-2876, 2019.

[28] W. Jiang, Y. Cui, B. Liu, W. Hu and Y. Xi, "A Dual-Band MIMO Antenna With Enhanced Isolation for 5G Smartphone Applications," IEEE Access, vol. 7, pp. 112554-112563, 2019.

[29] W. Jiang, B. Liu, Y. Cui and W. Hu, "High-Isolation Eight-Element MIMO Array for 5G Smartphone Applications," IEEE Access, vol. 7, pp. 34104-34112, 2019.

[30] Y. Wang, Y. Ban, Z. Nie and C. Sim, "Dual-Loop Antenna for 4G LTE MIMO Smart Glasses Applications," IEEE Antennas and Wireless Propagation Letters, vol. 18, no. 9, pp. 1818-1822, Sep. 2019.

[31] Kai-Kit Wong, R. D. Murch and K. B. Letaief, "Optimizing time and space MIMO antenna system for frequency selective fading channels," IEEE Journal on Selected Areas in Communications, vol. 19, no. 7, pp. 1395-1407, 2001.

[32] J. Akhtar and D. Gesbert, "Spatial multiplexing over correlated MIMO channels with a closed-form precoder," IEEE Transactions on Wireless Communications, vol. 4, no. 5, pp. 2400-2409, 2005.

[33] P. K. M. Ho, K. Yar and P. Y. Kam, "Cutoff Rate of MIMO Systems in Rayleigh Fading Channels With Imperfect CSIR and Finite Frame Error Probability," IEEE Transactions on Vehicular Technology, vol. 58, no. 7, pp. 3292-3300, 2009.

\section{BIOGRAPHIES OF AUTHORS}

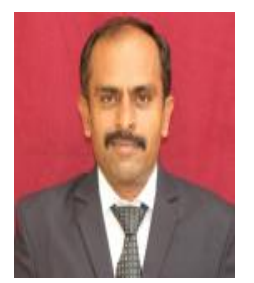

Shivapanchakshari T. G. is working as an Associate Professor in Cambridge Institute of Technology, Bangalore. He is pursuing his Ph.D. degree in Visvesvaraya Technological University, Belagavi, India. His fields of interest are Smart antennas, Electromagnetics and Digital Communication. He has published many papers on smart antennas \& OFDM systems.

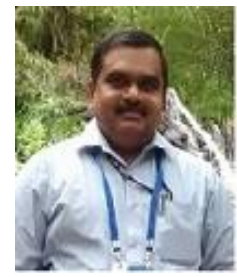

Dr. H. S. Aravinda, is a professor in the department of ECE in JSSATE, Bangalore. He has obtained his Ph D degree from Visvesvaraya Technological University, Belagavi, India. His fields of interest are Signal Processing and Communication. He has published many papers in reputed journals in the field of signal processing. Presently, he is guiding six research scholars. 